\section{Redeployed as a district nurse}

\author{
Lwazi Sibanda ${ }^{1}$ (pictured) with Vanessa Muirhead² reflects on being a dentist \\ redeployed as a district nurse during the COVID-19 pandemic.
}

${ }^{1}$ Clinical Leadership Fellow, Northamptonshire Healthcare NHS Foundation Trust, Salaried Primary Care Dental Service Headquarters, Willowbrook Health Centre, Cottingham Road, Corby, NN17 2UR: ${ }^{2}$ Centre of Dental Public Health and Primary Care, Institute of Dentistry, Queen Mary University of London, Turner Street, London, E1 2AD, UK

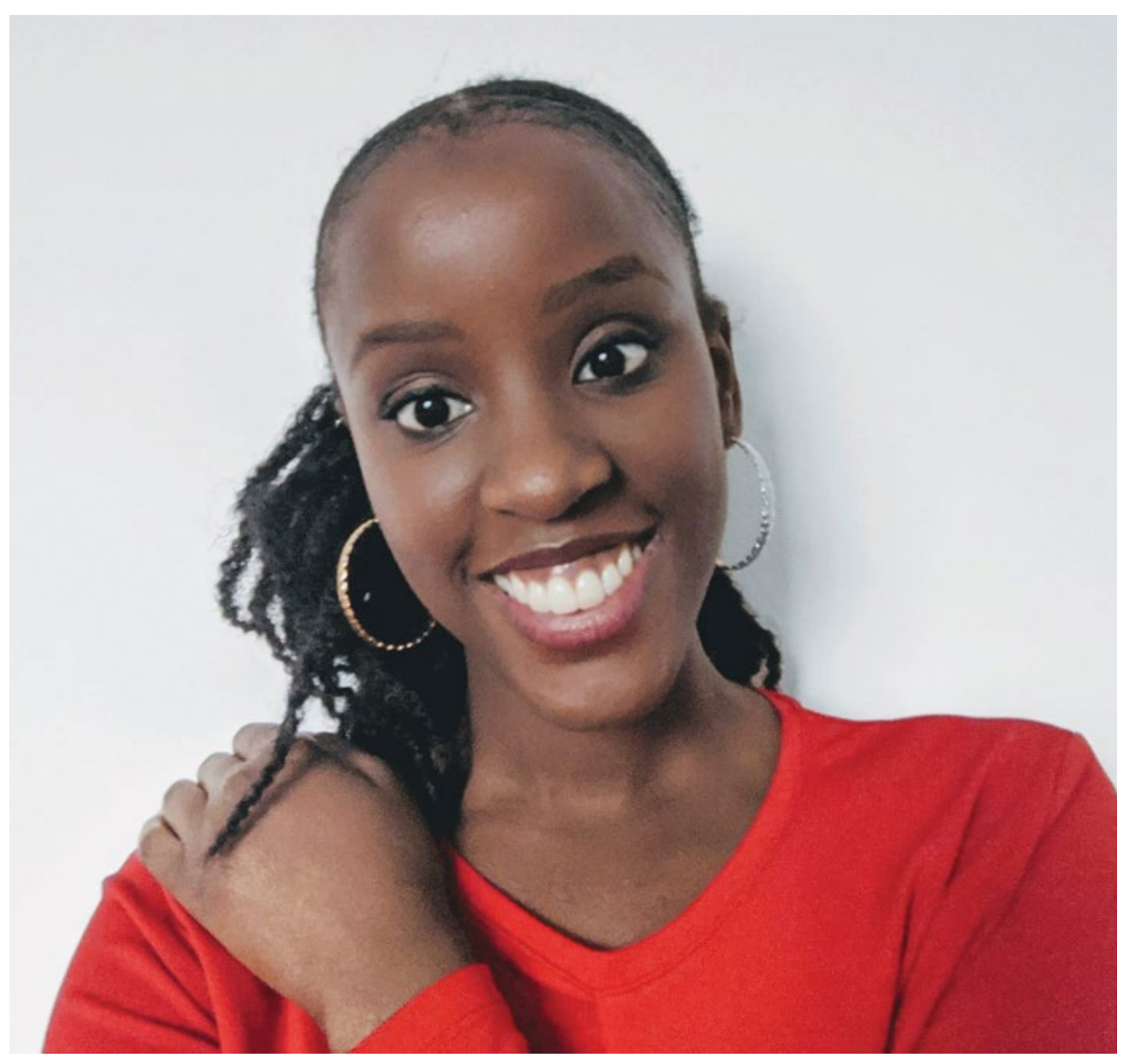

I nitially, I was apprehensive about the thought of being redeployed to the district nursing team while working as a dental core trainee (DCT). I was working as a DCT2 in paediatric dentistry, having previously held a DCT1 post in community dental services and oral and maxillofacial surgery (OMFS), as well as having worked in general practice. My first encounter with district nursing was through references I heard whilst working as an OMFS Senior House Officer, when I had to arrange for a patient to have their sutures removed in the community after their discharge. I was unfamiliar with the role that the district nursing team played. Despite any anxieties concerning my new role, I was aware of the great need for support for the NHS during the pandemic, as well as new pressures on the district nursing teams. With support from the dental team, I was fully supported to start my new role.

After undergoing training and completing competencies, I started my new role in the district nursing team. The team consisted of assistant practitioners, community staff nurses, health care assistants, as well as district nurses. Each member of the team competencies, working to support the whole team in the most effective way. This began with observing colleagues, followed by had their own unique role with different assisting and then independently visiting patients and delivering necessary care. My average day is shown in Box 1.

I provided care to patients in their homes, as well as in residential homes, with the majority of patients being housebound and often elderly. Throughout this time, I was able to apply medical knowledge from my dental training. This was applied through taking history when patients had additional complaints and raising this with a senior member of the team. I managed a patient with a low blood glucose reading, recorded and interpreted observations on a NEWS2 [National Early Warning Score] chart and used my knowledge of the aseptic technique from my clinical experience theatres. Wound care accounted for the majority of care for patients. I found that the management of chronic wounds often required primary and secondary dressings, as well as compression (if leg wounds). This was very different from the management of wounds that I had encountered during my OMFS experience, which usually consisted of lacerations that could be closed primarily using sutures. Moreover, the need for flexibility was crucial; this included being able to work in tight spaces in patients' houses.

Daily handover involved discussing the patients we had provided care for, as well as any new referrals or updates to patients. This provided an excellent opportunity to reflect on my assessment and treatment and learn from other members of the district nursing team. As I was working alone, handover served as a reminder that each home visit accounted for the patient's overall plan and a reminder each treatment played a significant part in the patient's comprehensive care.

The handover also provided a culture of celebration within the team. For example, this included celebrating patients gaining more independence with their care, as well as staff members completing competencies. Through presenting my patients during handover, I continued to grow in confidence.

My new role gave me insight into the patient pathway and access to the care provided by the district nursing team. This gave me an appreciation for the multiagency approach required by the district nursing team, and other community services, general medical practitioners, hospital 
